\title{
CONOCIMIENTOS Y PERCEPCIÓN DE ESTUDIANTES DE PSICOLOGÍA SOBRE EL TRABAJO PROFESIONAL EN PERSONAS CON VIH
}

\author{
Ricardo Sánchez Medina, María de los Ángeles Estrada Martínez, Consuelo Rubi Rosales Piña, \\ David Javier Enríquez Negrete y Maria Fernanda Lozano Quiroz \\ Universidad Nacional Autónoma de México \\ México
}

\begin{abstract}
RESUMEN
Los objetivos de la presente investigación fueron: 1) identificar el nivel de conocimientos acerca de VIH/SIDA, creencias y uso del condón en estudiantes de psicología, y 2) conocer su percepción cerca de VIH y del trabajo profesional con personas diagnosticadas con VIH. Por medio de un diseño transversal se evaluó a 439 estudiantes de Psicología, a quienes se les aplicó una batería de instrumentos de conocimientos respecto a VIH, ITS y uso de condón; creencias hacia el uso del condón, consistencia hacia el uso del condón, además de la estrategia de redes semánticas naturales modificadas para evaluar su percepción del VIH y del trabajo del psicólogo ante el VIH. Se encontró que tienen un nivel aceptable de conocimientos sobre VIH, pero no sobre el uso del condón; tienen creencias favorables hacia su uso, pero no implica que sean consistentes; tienen una valoración positiva hacia la atención de personas con $\mathrm{VIH}$. Se discute en términos de la importancia de formar a los estudiantes acerca de salud sexual y de cómo trabajar con personas diagnósticas con $\mathrm{VIH}$.
\end{abstract}

Palabras Clave:

estudiantes, VIH, aptitud, conocimientos, actitudes, práctica en salud

\section{KNOWLEDGE AND PERCEPTION OF PSYCHOLOGY STUDENTS ON PROFESSIONAL WORK WITH PEOPLE WITH HIV}

\begin{abstract}
The objectives of this research were: 1) identify the level of knowledge about HIV/AIDS, beliefs, and condom use in psychology students; and 2) know their perception about HIV and professional work with people diagnosed with HIV. Through a cross-sectional design, 439 psychology students were evaluated, to whom a battery of instruments on knowledge about HIV, STIs and condom use was applied; beliefs towards condom use, consistency towards condom use, in addition to the strategy of modified natural semantic networks to evaluate their perception of HIV and the work of the psychologist in dealing with HIV. They were found to have an acceptable level of knowledge about HIV, but not about condom use; have favorable beliefs towards their use, but it does not imply that they are consistent; They have a positive assessment of the care of people with HIV. It is discussed in terms of the importance of training students on sexual health and how to work with people diagnosed with HIV.
\end{abstract}

Keywords:

students, HIV, aptitude, knowledge, attitudes, practice in health

Bitácora del ARtículo:

| Recibido: 10 de Mayo de 2021 | Aceptado: 20 de septiembre de 2022 | Publicado en línea: Enero - Junio de 2022| 


\title{
Autoría y Derechos de Propiedad Intelectual
}

\section{CONOCIMIENTOS Y PERCEPCIÓN DE ESTUDIANTES DE PSICOLOGÍA SOBRE EL TRABAJO PROFESIONAL EN PERSONAS CON VIH}

\author{
Ricardo Sánchez Medina, María de los Ángeles Estrada Martínez, Consuelo Rubi Rosales Piña, \\ David Javier Enríquez Negrete y Maria Fernanda Lozano Quiroz \\ Universidad Nacional Autónoma de México \\ México
}

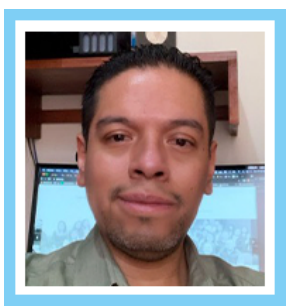

Ricardo Sánchez Medina FES- Iztacala, UNAM Correo: ricardo.sanchez@iztacala. unam.mx

Doctor en Psicología en la UNAM en el campo de Psicología y Salud. Acreditado como Tutor en Educación E-learnig por parte de la FESI-UNAM; cuenta con una especialidad en entornos virtuales de aprendizaje por la OEI. Profesor Titular B de tiempo completo definitivo en la FESI-UNAM, es tutor y docente de la Residencia en Terapia Familiar en el programa de maestría y doctorado la UNAM. Miembro del Sistema Nacional de Investigadores Nivel 1.

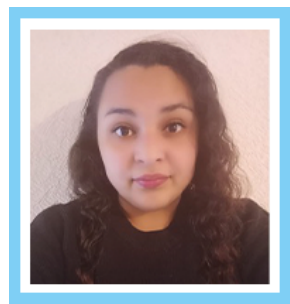

María de los Ángeles Estrada Martínez

FES- Iztacala, UNAM

Correo: angieestrada82@gmail.com

Licenciada en Psicología por la UNAM. Colaboradora en proyectos de investigación en el Laboratorio de Psicología, Tecnología y Salud (LPTS) perteneciente al Grupo de Investigación en Procesos Psicológicos y Sociales (GIPPS) adscrito a la Facultad de Estudios Superiores Iztacala. Ponente en congresos especializados nacionales y coautora de capítulos de libro especializados y memorias en extenso. Ha elaborado y aplicado cursos en línea y talleres en el ámbito educativo y de la salud.

\section{CONTRIBUCIÓN DE lOS AUTORES}

Ricardo Sánchez Medina es responsable de la idea original del tema, redacción del artículo, integración de la información, recolección y análisis de resultados | María de los Ángeles Estrada Martínez estuvo a cargo de la redacción del artículo, integración de la información, recolección y análisis de resultados | Consuelo Rubi Rosales Piña participó en la revisión del artículo, aplicación y recolección de la información y análisis de resultados | David Javier Enríquez Negrete colaboró en la búsqueda y análisis de información en bases de datos especializadas, análisis de resultados. | Maria Fernanda Lozano Quiroz participó en la búsqueda y análisis de información en bases de datos especializadas, análisis de resultados.

\section{AGRADECIMIENTOS}

Trabajo realizado con el apoyo del Programa UNAM-DGAPA-PAPIME PE302819

\section{Datos de FiLiación de los Autores}

Facultad de Estudios Superiores Iztacala, UNAM

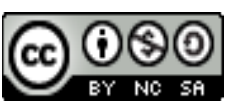

Copyright: ( 2022 Sánchez-Medina, R.; Estrada-Martínez, M.A.; Rosales-Piña, C.R.; Enríquez-Negrete, D.J; \& Lozano-Quiroz, M.F.

Este es un artículo de acceso abierto distribuido bajo los términos de la licencia Creative Commons Reconocimiento-NoComercial 4.0 Internacional, por lo que su contenido gráfico y escrito se puede compartir, copiar y redistribuir total o parcialmente sin necesidad de permiso expreso de sus autoras con la única condición de que no se puede usar con fines directamente comerciales y los términos legales de cualquier trabajo derivado deben ser los mismos que se expresan en la presente declaración. La única condición es que se cite la fuente con referencia a la Revista Digital Internacional de Psicología y Ciencia Social y a sus autoras. 


\section{TABLA DE CONTENIDO}

MÉTODO

Participantes, 26

Instrumentos, 26

Procedimiento, 27

Análisis de resultados, 27

RESULTADOS

Discusión

CONCLUSIONES

REFERENCIAS 
E I VIH aún es una de las infecciones con mayor estigma social debido a que su transmisión principal es por vía sexual; en el caso particular de México se habla de una epidemia concentrada, es decir, de los 9,828 nuevos casos que se presentaron durante 2019, la mayoría se encuentran en una población específica denominada vulnerable o de riesgo; siendo sobre todo hombres que tienen relaciones sexuales con hombres $(\mathrm{HSH})$, mujeres transexuales y trabajadoras sexuales (Centro Nacional para la Prevención y Control del VIH/Sida, 2019).

Hoy se sabe que los pacientes diagnosticados con $\mathrm{VIH}$ deben tomar medicamentos antirretrovirales para contrastar los efectos del virus; para ello es necesario que conozcan en qué consiste el que una persona adquiera el virus, así como las implicaciones hacia su salud; se espera que constantemente se hagan pruebas de $\mathrm{VIH}$ para conocer su estado serológico, y que además realicen cambios en su estilo de vida, como modificar su alimentación, hacer ejercicio, evitar el consumo de sustancias dañinas y adictivas, y reducir conductas sexuales de riesgo (Arias-Colmenero et al., 2020, Wondemagegn y Berkessa, 2020; Wray et al., 2020). Lo anterior es relevante porque estas condiciones son las mínimas necesarias para mantener una buena calidad de vida, para lo cual es necesario el acompañamiento de profesionales altamente capacitados.

Dado que la epidemia actual de VIH sigue sumando nuevos casos, es muy probable que los profesionales de la salud (médicos, enfermeras y psicólogos, entre otros) atiendan en su vida laboral al menos a una persona con alto riesgo de contraer $\mathrm{VIH}$ o con un diagnóstico de éste (Peer, de Villiers, Jonathan, Kalombo y Kengne, 2020), por lo cual se espera que dicha atención esté libre de cualquier prejuicio que influya en la atención (Shahar, Maor y Moshe-Eilon, 2020).

Estos prejuicios generalmente se asocian al estigma del VIH/SIDA, los cuales fomentan actitudes negativas ante las personas con diagnóstico, lo que podría generar una barrera para la promoción y el mantenimiento de la salud, presentando dificultades para el acceso o mantenimiento del tratamiento (Altice, Evuarherhe, Shina, Carter y Beaubrun, 2019; Damulira et al., 2019). Por lo anterior, es importante que los profesionales de la salud sean capaces de guiar a los usuarios de una manera ética y profesional que esté libre de estos prejuicios.

De acuerdo con Chamorro y Betanco (2019), Sigüenza y Solano (2017), y Uribe y Orcasita (2011), los profesionales de la salud son los principales agentes que proporcionan información referente a VIH/SIDA, así como las implicaciones que traerá a su vida la infección, de manera que pueden ofrecer un tratamiento que permita mejorar la calidad de vida y la adherencia al tratamiento en personas con VIH (Altice et al., 2019; Ballester-Arnal et al., 2016).

En este sentido, se han hecho investigaciones orientadas a identificar qué saben los profesionales de la salud acerca del VIH y cuál es su actitud ante la infección que permiten tener indicadores que favorecen o entorpecen la atención; en términos generales se ha observado que el nivel de conocimientos entre los profesionales de la salud (médicos, enfermeros, farmacia, trabajadores sociales) es de un nivel medio-alto respecto a sus características, formas de transmisión y sintomatología (Sigüenza y Solano, 2017). Sin embargo, no basta que los profesionales de la salud cuenten con buenos niveles de información, sino que también se espera que puedan tener actitudes y creencias favorables en la atención de personas con $\mathrm{VIH}$, porque se ha encontrado que es un aspecto importante que se relaciona con la efectividad en las intervenciones (Pittenger et al., 2019; Wagner, McShane, Hart y Margolese, 2016); en cambio, cuando tienen conocimientos erróneos, creencias negativas o una mala percepción ante el VIH repercute en el trato hacia las personas con $\mathrm{VIH}$, en el cual se brinda una atención deficiente y con temor (Peer et al., 2020; Mak, Cheng, Law, Cheng y Chan, 2015; Marshall, Brewington, Kathryn, Haynes y Zaller, 2017).

Por ejemplo, Ebrahimi, Sindarreh y Nasirian (2020) encontraron que el personal de salud, como enfermeros, dentistas, laboratoristas y administrativos, entre otros, tienen una actitud frente al VIH no muy satisfactoria, lo cual pudiera relacionarse con el estigma y discriminación, ya sea para negar la atención, clasificarlos como culpables o victimizarlos (Feyissa, Lockwood, Woldie y Munn, 2019; Nguyen, Go, Bui, Gaynes y Pence, 2019; Varas-Díaz et al., 2019).

En otros estudios se ha reportado que el personal de salud, enfermeros y médicos, consideran que no están capacitados para el trabajo con la población con VIH, y existe el temor de contraer el virus por exposición accidental ocupacional (Conejeros, Emig, Ferrer, Cabieses y Acosta, 2010; Valdez, Vargas y Apaza, 2010). En consecuencia, es evidente la necesidad de incluir en etapas tempranas a nivel curricular materias que no sólo se enfoquen en los conocimientos y habilidades, sino también en sus actitudes, como la percepción de la atención de personas con VIH y de qué manera podrían impactar en la atención (Mascolo et al., 2011). 
Un aspecto importante a considerar es que en general la evaluación de estos conocimientos y actitudes se enfoca en médicos, enfermeros, personal de laboratorio y farmacia (Sigüenza y Solano, 2017), o incluso en estudiantes en estas áreas de formación (Rodríguez, 2019). Si bien en el trabajo con personas con $\mathrm{VIH}$ se requiere una labor multidisciplinaria (Llibre, Fuster-Ruizdeapodaca, Rivero y Fernández, 2018), es necesario considerar el papel del psicólogo, en términos de su contribución para mejorar la calidad de vida y la adherencia al tratamiento, así como en el cuidado de la salud emocional (Canales-Zavala et al., 2019; Finkelstein-Fox, Park y Kalichman, 2020; Shey, Dzemo, Siysi, Ekobo y Jelil, 2020).

Algunas investigaciones que han evaluado a estudiantes de Psicología indican resultados similares con otros profesionales, ya que también se observa que tienen un nivel de conocimiento acerca del virus medio-alto (Fernández et al., 2013), aunque se ha informado que no tienen una actitud favorable hacia los pacientes con VIH (Pineda-Roa y Campo-Arias, 2018), y no se identificaron estudios que valoraran la actitud hacia el trabajo profesional con personas con $\mathrm{VIH}$.

De acuerdo con Sharma, Mudgal, Thakur, Gaur y Aggarwal (2020), tener conocimientos de salud no es suficiente para garantizar que los mismos profesionales de la salud tengan una actitud favorable y sigan un estilo de vida saludable; esta congruencia es relevante si se espera que ellos sean quienes promuevan la salud y prevengan enfermedades, por lo que es necesario que usen condón en sus relaciones sexuales (Novales-Castro, Amato y Hurtado, 2019).

De manera que es evidente la necesidad de contar con psicólogos capaces de ofrecer atención a las personas diagnosticadas con VIH de manera ética y profesional, y que al mismo tiempo sean capaces de cuidar de su salud sexual; todo ello implica que durante su formación adquieran conocimientos respecto a los aportes de la Psicología de la Salud, del VIH, actitudes y percepciones positivas hacia el $\mathrm{VIH}$, atención de personas diagnosticadas con este virus, así como el trabajo personal hacia la salud sexual que permitan, en colaboración con otros profesionales, una atención integral libre de prejuicios hacia las personas con $\mathrm{VIH}$.

Si bien es un trabajo complejo, en esta primera etapa de la presente investigación se pretende identificar lo que saben los estudiantes de Psicología acerca del VIH, su conducta sexual y la percepción que tienen de la atención de personas diagnosticadas con este virus, que permita el diseño de programas de intervención orientados a ello; en una segunda etapa, se espera identificar los conocimientos y habilidades que debe tener un psicólogo en la atención de personas con $\mathrm{VIH}$, de modo que se pueda integrar en su formación, y que se impacte en una mejor atención por parte de los futuros profesionales de la Psicología.

Con base en lo anterior, los objetivos de esta investigación son 1) identificar el nivel de conocimientos acerca de VIH/SIDA, creencias hacia el uso del condón, y su relación con el uso del condón en estudiantes de Psicología, y 2) conocer su percepción respecto del $\mathrm{VIH}$ y del trabajo profesional con personas diagnosticadas con $\mathrm{VIH}$.

\section{Método}

\section{Participantes}

439 estudiantes de Psicología de un sistema en línea de una universidad pública; $83.4 \%$ mujeres y $16.6 \%$ hombres; edad promedio de 33.15 años (SD = 8.96); respecto al semestre que cursaban al momento de responder, está distribuido de la siguiente manera: primer semestre (8.40\%), segundo semestre $(5.85 \%)$, tercer semestre $(9.16 \%)$, cuarto semestre $(9.67 \%)$, quinto semestre $(4.58 \%)$, sexto semestre $(4.07 \%)$, séptimo semestre $(6.62 \%)$, octavo semestre $(34.35 \%)$ y noveno semestre $(17.30 \%)$. Mediante un muestreo no probabilístico de tipo intencionado (Kerlinger y Lee, 2002) se invitó a los estudiantes de Psicología a tomar un curso en línea enfocado en la atención de personas con $\mathrm{VIH}$, y cuyo requisito era que, antes de iniciarlo, respondieran una encuesta acerca de lo que conocían, pensaban y hacían en torno al VIH y a su conducta sexual. Como criterio de exclusión se consideró a los estudiantes que no respondieron toda la encuesta.

\section{Instrumentos}

Prueba de conocimientos sobre VIH/SIDA e ITS. Se aplicó una versión corta de la prueba validada por Robles y Díaz-Loving (2011) en estudiantes universitarios. La prueba comprendía 12 reactivos divididos en cuatro áreas: características de ITS, prevención, transmisión y sintomatología del VIH/SIDA. Las opciones de respuesta fueron cierto, falso y no sé; con base en los resultados otorgados se obtuvo el porcentaje de respuestas correctas.

Prueba de conocimientos sobre el uso del condón. Se aplicó una versión corta de la prueba validada por Robles y Díaz-Loving (2011) en estudiantes universitarios. La prueba englobaba seis reactivos acerca de lo que saben del uso del condón en relaciones sexuales (como "Cuando se coloca un condón, es importante que se ajuste perfectamente, sin dejar espacio en la punta"). Las opciones de respuesta fueron cierto, falso y no sé; 
con base en los resultados otorgados se obtuvo el porcentaje de respuestas correctas.

Escala de creencias hacia el uso del condón. Consta de 12 reactivos que evalúan las creencias acerca del uso del condón (validada en estudiantes universitarios por Robles y Díaz-Loving, 2011, alfa de 0.84). Cuatro reactivos evalúan creencias negativas (como "Disminuye el placer sexual") y ocho evalúan creencias positivas (como "Podría hacer que te preocupes menos") respecto al uso del condón. Las opciones de respuesta están en formato tipo likert, con cinco opciones de respuesta que van de totalmente en desacuerdo a totalmente de acuerdo.

Consistencia en el uso del condón. Con base en la propuesta de DiClemente y Wingood (1995) se presentaron dos reactivos para evaluar el número de veces que tuvieron relaciones sexuales y usaron condón en los últimos tres meses. Con los resultados reportados se obtiene un índice de consistencia con valores entre cero (0) y uno (1), que es el resultado de dividir el número de veces de uso de condón en tres meses entre el número de relaciones sexuales, en ese mismo periodo de tiempo. Valores iguales a 1 indican que la persona es consistente en el uso del condón.

Percepción del VIH. Mediante la estrategia de redes semánticas naturales modificadas (RSNM) de Reyes-Lagunes (1993) se presentaron dos estímulos con los que se pedía a los participantes que mencionaran palabras que relacionan con los estímulos 1) VIH y yo, y 2) una persona con VIH es; luego se les pedía que las numeraran en orden de importancia, asignando el valor 1 a la que consideran más relevante, y así sucesivamente.

Percepción del trabajo del psicólogo ante el VIH. Con base en la estrategia de RSNM y en lo ya mencionado se presentaron dos estímulos: 1) trabajar con personas con $\mathrm{VIH}, \mathrm{y}$ 2) que se necesita para trabajar con personas con $\mathrm{VIH}$.

\section{Procedimiento}

Esta investigación tiene un enfoque cuantitativo y un diseño no experimental de corte transversal. Mediante una convocatoria se invitó a estudiantes de Psicología a un curso extracurricular en línea. El curso fue gratuito y se les mencionó que para poder cursarlo era necesario ser estudiante de Psicología y que al inicio respondieran una encuesta. A quienes aceptaron, al comienzo del curso se les mostró la encuesta; en ella se indicó que la información proporcionada sería confidencial y anónima, y que sólo sería utilizada con fines estadísticos. Cuando los estudiantes respondieron la encuesta tuvieron acceso a todo el curso, y quienes cumplieron los requisitos de aprobación obtuvieron su constancia de asistencia.

\section{Análisis de resultados}

A través del paquete estadístico SPSS v26 se presentan datos descriptivos de los resultados obtenidos por las pruebas de conocimientos, creencias y consistencia en el uso del condón; después, con una $r$ de Pearson se correlacionaron los índices obtenidos. Para el análisis de las RSNM se utilizaron hojas de cálculo de Excel, con las que se obtuvieron las frecuencias y ponderaciones de las palabras proporcionadas por los participantes; con base en el punto de corte de Catell se seleccionaron las palabras con mayor peso semántico.

\section{Resultados}

En la tabla 1 se muestran los estadísticos descriptivos de las variables evaluadas para cubrir el primer objetivo de la investigación; grosso modo se observa que tienen un nivel de conocimientos de sintomatología de VIH superior a $95 \%$ de respuestas correctas, y conocimientos entre 83 y $85 \%$ de respuestas correctas de transmisión, prevención y características de $\mathrm{VIH}$; sin embargo, también se encontró que tienen casi $60 \%$ de respuestas correctas respecto al uso de condón en relaciones sexuales.

Respecto a las creencias acerca del uso del condón en relaciones sexuales, es necesario referir que la media teórica es 3, por lo cual la media obtenida superior a dicho número indica que tienen la creencia, y un valor menor a 3 indica que no la tienen; como se muestra en la tabla 1, los estudiantes de Psicología reportan creencias favorables hacia su uso; si bien la media de las creencias negativas indica que tienen poco este tipo de creencias, al observar los valores mínimos y máximos es evidente que los datos varían entre uno y cinco, lo cual indica que hay estudiantes que en sus respuestas aceptaron estar de acuerdo o en desacuerdo con dichas creencias, como que el usar condón en relaciones sexuales disminuye el placer sexual.

Por último, respecto a la consistencia en el uso del condón, en esta misma tabla se observa una media de 0.35 , lo cual indica que no usan de manera consistente el condón en sus relaciones sexuales, si se considera que los valores oscilan entre 0 (cero) y 1 (uno), en donde 0 significa que nunca usaron condón en sus relaciones sexuales en los últimos tres meses, y 1 indica que usaron condón en todas y cada una de las relaciones sexuales; es necesario indicar que del total de los participantes sólo $9.8 \%$ reportaron ser consistentes en el uso del condón, $1.4 \%$ que nunca lo usaron, y el resto $(88.8 \%)$ fueron inconsistentes en su uso.

En la tabla 2 se muestra un análisis de relación entre la consistencia en el uso del condón y el índice de 
Tabla 1.

Estadísticos descriptivos de conocimientos de VIH y uso de condón, creencias y uso consistente del condón en estudiantes universitarios.

\begin{tabular}{lccccccc} 
& MediA & SD & Mínimo & MÁximo & Asimetría & CuRtosis \\
\hline & \multicolumn{2}{c}{ Conocimientos } & & & & \\
\hline Sintomatología del VIH & 95.36 & 11.97 & 33.33 & 100 & -238 & 4.68 \\
\hline Transmisión del VIH & 83.06 & 24.34 & 0 & 100 & -1.20 & 0.44 \\
\hline Prevención del VIH & 84.28 & 19.46 & 33.33 & 100 & -0.80 & -0.34 \\
\hline Características de ITS & 85.19 & 17.90 & 33.33 & 100 & -0.62 & -0.81 \\
\hline Uso del condón & 61.19 & 21.58 & 0 & 100 & -0.24 & -0.26 \\
\hline Positivas & Creencias hacia el uso del condón & & & \\
\hline Negativas & 4.84 & 0.38 & 3 & 5 & -2.06 & 3.03 \\
\hline Consistencia del uso del condón & 2.01 & 1.10 & 1 & 5 & 0.81 & -0.14 \\
\hline
\end{tabular}

respuestas correctas en conocimientos, así como con el nivel obtenido en creencias hacia el uso del condón; los resultados indican que cuando los estudiantes tienen una puntuación alta en la consistencia en el uso del condón, tienen un nivel alto de conocimientos de la prevención de VIH (correlación positiva), y tienen índices bajos en las creencias negativas respecto al uso del condón en relaciones sexuales (correlación negativa).

Además se presentan las correlaciones entre las variables, y se observa de manera general que a mayor nivel de conocimientos en un área hay mayor nivel de conocimientos en otra área (correlaciones positivas), con excepción de los conocimientos de síntomas de $\mathrm{VIH}$; las creencias positivas se relacionaron con los conocimientos de prevención de VIH y del uso del condón (correlación positiva), mientras que para las creencias

negativas se encontró una correlación negativa con los conocimientos de prevención de VIH y con las creencias positivas. Es necesario comentar que todas las correlaciones significativas son medio-bajas (0.41 a 0.60) o bajas $(-0.40)$.

Respecto al segundo objetivo, en la figura 1 se muestran las principales palabras que atribuyen los estudiantes de Psicología al estímulo "El VIH y yo"; con base en el peso semántico que le atribuyen se observa que las palabras cercanas a 0 tienen mayor significado para los participantes, y conforme se va alejando lo va perdiendo. Lo anterior permite observar que las principales palabras que le otorgan es "enfermedad", "prevención" y "protección"; grosso modo le atribuyen aspectos relacionados con la prevención del VIH, estar informados acerca de VIH y el uso de condón; aunque

Tabla 2.

Correlación entre conocimientos de $\mathrm{VIH}$ y uso de condón, creencias y uso consistente del condón en estudiantes universitarios.

$\begin{array}{llllllll}1 & 2 & 3 & 4 & 5 & 6 & 7 & 8\end{array}$

1. Consistencia del uso del condón

2. Conocimientos: Sintomatología del VIH

0.015

3. Conocimientos: Transmisión del VIH

$0.022 \quad 0.035$

4. Conocimientos: Prevención del VIH

$0.155^{*} \quad 0.074 \quad 0.486^{* *}$

5. Conocimientos: Características de ITS

$0.0310 .153^{* *} \quad 0.250^{* *}$

6. Conocimientos: Uso del condón

0.137

0.088

$0.405^{* *}$

$0.306^{* *}$

7. Creencias positivas hacia el uso del condón

$\begin{array}{lllll}0.066 & 0.050 & 0.069 & 0.133 * * & 0.067\end{array}$

$-0.278^{* *}-0.027-0.060$

$-0.104^{*}$

$-0.024$

$0.098^{*}$

8. Creencias negativas hacia el uso del condón

* La correlación es significativa en el nivel 0.05 .

** La correlación es significativa en el nivel 0.01. 
también mencionan aspectos negativos como el miedo, el peligro y considerar que es mortal.

\section{Figura 1.}

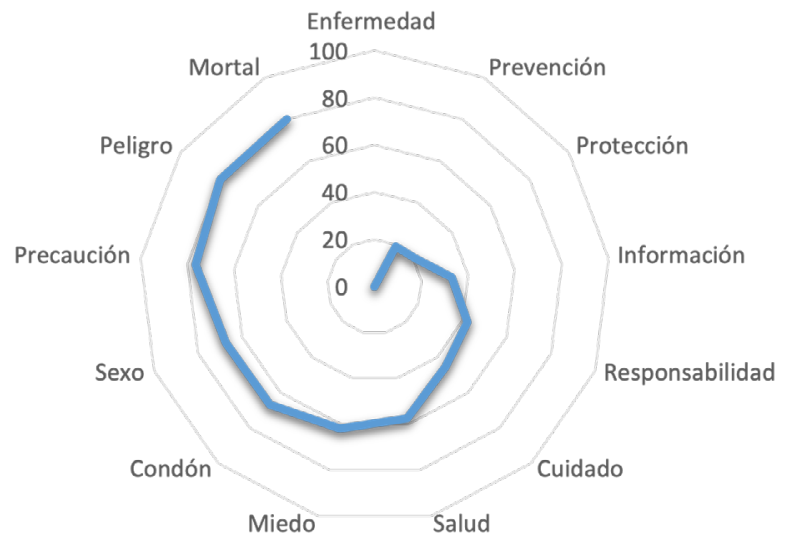

Principales palabras otorgadas por los estudiantes de Psicología al estímulo "EI VIH y yo".

Respecto de lo que piensan de una persona con $\mathrm{VIH}$, en la figura 2 se muestra que los participantes consideran que es una persona "normal", "enferma" e "igual"; mencionan aspectos positivos como "fuerte", "capaz" y "valiosa", y aspectos negativos como "vulnerable" y "discriminada".

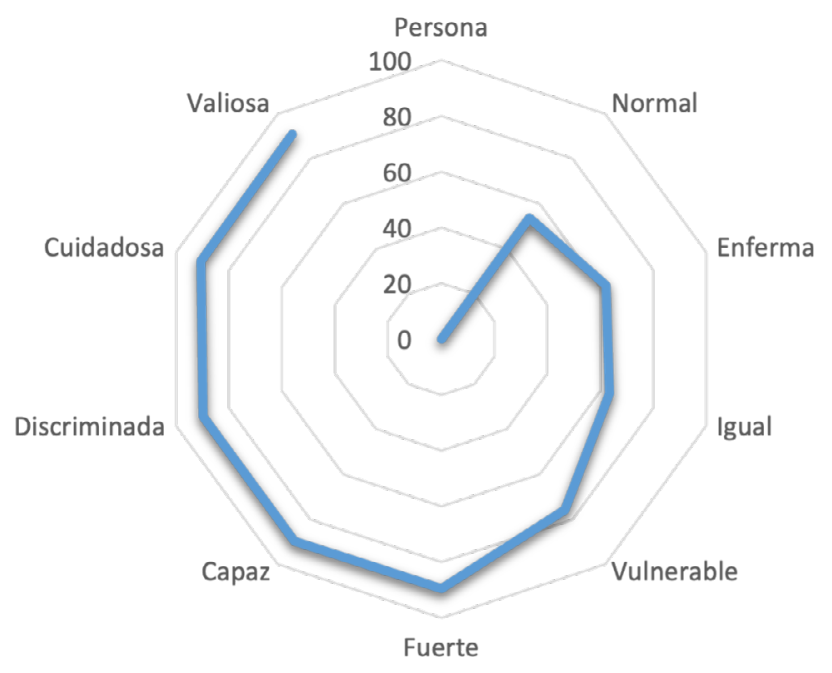

\section{Figura 2.}

Principales palabras otorgadas por los estudiantes de Psicología al estímulo "Una persona con VIH es...".

En la figura 3 se muestran las palabras que se relacionan con el trabajo profesional con personas que padecen VIH; los participantes mencionaron que necesitan ser empáticos, tener información del tema, ser respetuosos, tener cuidado, ser comprensivos, que sería algo normal, y que requiere aprendizaje y prevención.

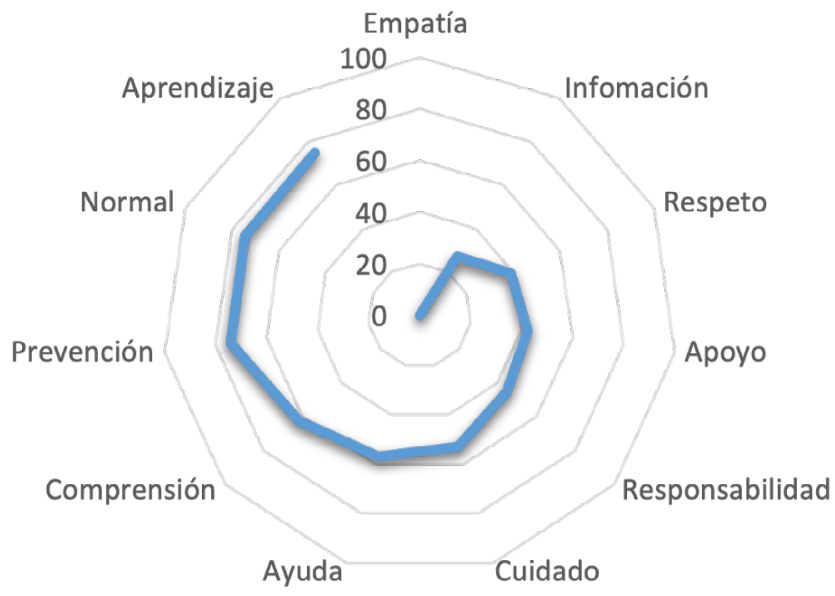

Figura 3.

Principales palabras otorgadas por los estudiantes de Psicología al estímulo "Trabajar con personas con VIH"..

Por último, en la figura 4 se muestran las palabras que los participantes relacionaron con el estímulo necesario para trabajar con personas con $\mathrm{VIH}$, las cuales se asocian con la información y la formación en el área, y habilidades actitudinales como empatía, respeto y comprensión, entre otras; además de tener interés en trabajar con el tema, ser tolerantes y tener apertura.

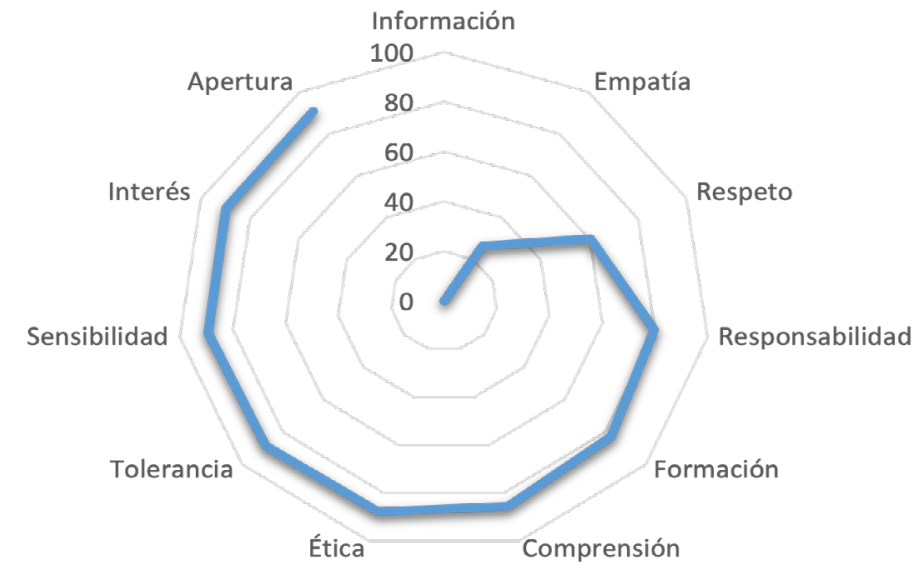

Figura 4.

Principales palabras otorgadas por los estudiantes de Psicología al estímulo "Qué se necesita para trabajar con personas con VIH".

\section{Discusión}

Los objetivos esta investigación fueron 1) identificar el nivel de conocimientos acerca de VIH/SIDA, creencias hacia el uso del condón, y su relación con el uso del condón en estudiantes de Psicología, y 2) conocer su percepción respecto al $\mathrm{VIH}$ y del trabajo profesional con personas diagnosticadas con VIH. 
Referente al primer objetivo, se encontró que los estudiantes de Psicología tienen un nivel alto de conocimientos de sintomatología, transmisión y prevención de $\mathrm{VIH}$, así como de las características generales de VIH, aunque se observa que sólo en los síntomas tuvieron más de $95 \%$ de respuestas correctas, y en las otras áreas entre 83 y $85 \%$; esto indica que están informados acerca del tema, pero es necesario identificar en qué áreas es necesario aumentar sus conocimientos para que tengan la información mínima necesaria al momento de trabajar con personas con VIH (Fernández et al., 2013; Mascolo et al., 2011), ya que, como se mencionó, es probable que sean el primer contacto que tienen las personas recién infectadas por $\mathrm{VIH}$.

Respecto a los conocimientos del uso del condón en relaciones sexuales, es preocupante que en general tuvieron una media de $60 \%$ de respuestas correctas; además, al revisar los valores mínimos y máximos hubo participantes que tuvieron $0 \%$ de respuestas correctas; esto es un área de oportunidad si se considera que los estudiantes conozcan qué deben hacer para evitar que el condón se rompa o se deslice durante el encuentro sexual, como que no deben usar condón femenino y masculino al mismo tiempo, el tipo de lubricante que puedan usar o cerca de la fecha de caducidad (Robles y Díaz-Loving, 2011). Si bien se encuentra en la literatura que la información es necesaria, pero no suficiente, para lograr cambios en el comportamiento, sí se requiere como primer paso en el establecimiento de comportamientos preventivos (Guerra, Aldana y Rojas, 2020). Lo anterior es relevante si se considera la necesidad de congruencia entre lo que hace el profesional y lo que transmite (Sharma et al., 2020), por lo cual no basta que los futuros profesionales de Psicología sepan qué es el VIH, sino que al mismo tiempo tengan una conducta sexual segura, por lo que en esta investigación se buscó analizar sus creencias y el uso del condón en relaciones sexuales en los estudiantes.

En lo que se refiere a las creencias hacia el uso del condón, se observa que los estudiantes de Psicología consideran que usar condón en relaciones sexuales es bueno para ellos (creencias positivas), respecto a las creencias negativas, si bien la media del grupo estuvo por debajo de la media teórica (3), esto indicaría que no están de acuerdo con las creencias de que el condón es malo en las relaciones sexuales; se aprecia que hay estudiantes con índices de 1 y de 5, lo cual indica que hay personas que sí tienen estas creencias negativas, siendo un punto indispensable a considerar respecto a su importancia del uso del condón en relaciones sexuales (Bolaños, 2019; Gredig, Le Breton, Granados y Solís, 2020).
Respecto al uso del condón en todas y cada una de sus relaciones sexuales, se observa que son pocos los estudiantes consistentes en el uso del condón, lo cual es un aspecto importante para tener en cuenta si se considera que la única manera de prevenir el $\mathrm{VIH}$ en personas sexualmente activas es el uso correcto y constante del condón (Elshiekh, Hoving y De Vries, 2020).

Para corroborar lo anterior, se relacionó la consistencia en el uso del condón con los conocimientos de VIH y creencias; se encontró que lo que saben de prevención de $\mathrm{VIH}$ y no tener creencias negativas se asoció positivamente con el uso del condón en relaciones sexuales (Robles y Díaz-Loving, 2011).

Lo anterior se torna relevante en los estudiantes de Psicología por dos motivos: 1) porque es necesario diseñar estrategias de intervención para promover el uso del condón en relaciones sexuales, ya que al menos en la muestra evaluada se observa que tienen conductas de riesgo que los puede hacer susceptibles de infección por VIH (Novales-Castro et al., 2019), y 2) de acuerdo con Sharma et al. (2020), es indispensable que como profesionales de la salud se adopten estilos de vida saludables acordes con lo que se desea promover; en este sentido, no basta con que los estudiantes de Psicología estén formados y capacitados en la prevención o tratamiento del $\mathrm{VIH}$, sino que además tengan conductas sexuales preventivas, creencias positivas hacia el uso del condón, así como una actitud favorable hacia el trabajo profesional con personas con diagnóstico de $\mathrm{VIH}$.

El segundo objetivo estuvo orientado hacia las actitudes y creencias que tienen los estudiantes de Psicología hacia el VIH, así como el trabajo profesional con personas con VIH. En primera instancia se analizó qué instrumento podría utilizarse que fuese culturalmente válido y con propiedades psicométricas adecuadas (Reyes-Lagunes, 1993); en dicha revisión se encontraron instrumentos que utilizan diversos profesionales, sobre todo en médicos y enfermeros (Conejeros, et al., 2010; Uribe y Orcasita, 2011); además, de acuerdo con Pineda-Roa y Campo-Arias (2018), si bien las actitudes frente al VIH no han variado mucho en los últimos años, en términos de su evaluación, no se ha encontrado un instrumento con buenas propiedades psicométricas, como lo reportan en su investigación acerca de la evidencia empírica que presentan de una escala de actitudes referentes al VIH/SIDA.

Así que se optó por utilizar la estrategia de RSNM que, de acuerdo con Reyes-Lagunes (1993), se puede conocer el significado psicológico de uno o varios conceptos e identificar las conductas que las personas consideran tiene el constructo de interés, y que ha sido utilizada en la evaluación de una gran diversidad de constructos y 
ha mostrado evidencia empírica del aporte en la definición de constructos (Chávez, Zapata, Petrzelová y Villanueva, 2018; Flores-Cano, Reyes-Lagunes, Rivera-Aragón y Mansilla-Corona, 2020; Granados, 2019).

En el caso particular de los objetivos de la investigación se utilizó para conocer lo que piensan los estudiantes de Psicología acerca del VIH, y del trabajo profesional con VIH. En la primera parte se observa que los participantes asociaron palabras al VIH enfocadas en que es una enfermedad, que es mortal y el peligro que puede implicar, pero también aspectos de prevención, información y responsabilidad; mencionaron dos conductas relacionadas con sexo y condón, y una emoción enfocada en el miedo. Grosso modo se distingue que en la muestra evaluada se presentan tanto valoraciones positivas como negativas, lo cual pudiera tener un impacto en su conducta sexual (Ebrahimi et al., 2020; Elshiekh, Hoving y De Vries, 2020), por lo que se considera necesario en futuras investigaciones analizar cómo estos significados se relacionan con su vida sexual e incluso con la presencia de problemas de salud sexual.

En el estímulo "una persona con VIH es", mencionaron palabras descriptivas, positivas y negativas, primero señalando que es "persona", "normal" e "igual"; segundo mencionando "fuerte", "capaz", "cuidadosa" y "valiosa"; y por último "enferma", "vulnerable" y "discriminada". De manera general las palabras descriptivas ocuparon los primeros lugares, esto podría deberse a que los participantes pueden tratar de ser neutrales al momento de definir a una persona con VIH (Feyissa et al., 2019; Mak et al., 2015). Es necesario atender si esta valoración negativa de los estudiantes es algo que perciben por parte de la sociedad, o si es la manera en que ellos lo perciben, de manera que se pueda tener un indicador que permita diseñar estrategias de intervención para, por un lado, modificar las creencias de los estudiantes de Psicología y, por otro lado, para que puedan cambiar estas creencias en la población en general.

Cuando se les preguntó acerca del trabajo y qué se necesita para trabajar con personas con $\mathrm{VIH}$, dentro de las principales palabras que los estudiantes reportaron están ser empáticos, tener información y ser respetuosos; esto es relevante si se considera que son habilidades actitudinales que deben poseer los psicólogos en su quehacer profesional, y más aún tratándose de personas con $\mathrm{VIH}$, porque se ha encontrado que las actitudes del profesional influyen en la eficacia del tratamiento (Marshall et al., 2017; Peer et al., 2020).

\section{Conclusiones}

Todo profesional de la salud debe tener una congruencia entre lo que hace y lo que transmite, y debe llevarse a cabo desde su formación. En el caso particular de la atención de personas con $\mathrm{VIH}$, hay algunas creencias respecto a ella que podrían dificultar la intervención psicológica hacia este sector de la población; si bien es necesario un trabajo multidisciplinario, en el caso de la Psicología no es un área muy explorada en comparación con médicos y enfermeros, por lo cual es necesario generar investigación al respecto.

Con base en los resultados obtenidos se puede decir que la muestra evaluada tiene conocimientos adecuados de $\mathrm{VIH}$, pero no del uso del condón, lo cual pude tener un impacto en su salud sexual y en la posible atención que pudieran brindar a personas con $\mathrm{VIH}$. Si bien tienen creencias favorables hacia su uso, no necesariamente se asocia con la conducta sexual de los estudiantes, en particular sobre usarlo en todas y cada una de sus relaciones sexuales; en este sentido es necesario un entrenamiento en habilidades para promover el uso correcto y consistente del condón (Fernández et al., 2013; Sharma et al., 2020), para que los universitarios puedan tener un comportamiento sexual seguro y eviten problemas de salud sexual, como lo es la infección por VIH (Novales-Castro et al., 2019; Robles y Díaz-Loving, 2011).

Respecto a la percepción del trabajo profesional, no se encontraron instrumentos para su evaluación; sin embargo la estrategia de RSNM permitió tener un panorama general de la percepción de los estudiantes de Psicología; los resultados mostraron que tienen una valoración positiva hacia intervenir con personas con $\mathrm{VIH}$; como ya se mencionó, el siguiente paso es identificar qué habilidades profesionales deberían tener y con cuáles cuentan para que puedan brindar atención con una actitud ética y profesional (Pittenger et al., 2019).

Si bien estos resultados ofrecen un panorama general de los conocimientos que tienen acerca del $\mathrm{VIH}$ y el condón, de las creencias relacionadas con el uso del condón y de la percepción del trabajo profesional con personas diagnósticas por este virus, es necesario destacar que es indispensable ampliar la muestra para saber si sucede lo mismo o qué diferencias hay.

Por otro lado, no se contó con instrumento válido y confiable para evaluar las actitudes hacia el trabajo profesional con personas con VIH, por lo que se decidió utilizar la estrategia de RSNM, de manera que es una aproximación que permite indagar acerca de sus percepciones; además, los resultados obtenidos permitirán el diseño de un instrumento de evaluación para tener un instrumento culturalmente válido en estudiantes de 
Psicología y poder evaluar sus actitudes hacia el trabajo profesional con personas diagnosticadas con $\mathrm{VIH}$.

A lo largo del trabajo se menciona la importancia del desarrollo de habilidades por parte de los estudiantes de Psicología en la atención del VIH; sin embargo, en esta investigación no se delimitó qué habilidades son las que deben tener ni se evaluó cuáles de esas habilidades tienen; según se indicó, esta es una segunda etapa de la investigación en que se buscará identificar y evaluarlas para de esta manera hacer una evaluación completa de la atención integral que debe tener todo psicólogo en la atención de sujetos con VIH, y que dicha evaluación sirva como una evaluación diagnóstica en el diseño de programas de formación.

\section{Referencias}

Altice, F., Evuarherhe, O., Shina, S., Carter, G., \& Beaubrun, A. C. (2019). Adherence to HIV treatment regimens: Systematic literature review and meta-analysis. Patient Preference \& Adherence, 13, 475-490. https://doi.org/10.2147/PPA. S192735

Arias-Colmenero, T., Pérez-Morente, M. Á., Ramos-Morcillo, A. J., Capilla-Díaz, C., Ruzafa-Martínez, M., \& HuesoMontoro, C. (2020). Experiences and attitudes of people with HIV/AIDS: A systematic review of qualitative studies. International Journal of Environmental Research and Public Health, 17(2), 639-653. https://doi.org/10.3390/ ijerph17020639

Ballester-Arnal, R., Gómez-Martínez, S., Fumaz, C. R., GonzálezGarcía, M., Remor, E., \& Fuster, M. J. (2016). A spanish study on psychological predictors of quality of life in people with HIV. AIDS Behavior, 20, 281-291. https://doi. org/10.1007/s10461-015-1208-6

Bolaños, M. (2019). Barreras para el acceso y el uso del condón desde la perspectiva de género. Horizonte Sanitario, 18(1), 67-74. https://doi.org/10.19136/hs.a18n1.2306

Canales-Zavala, M. A., Hernández-Ruiz, A. V., González-Venegas, S., Monroy-Avalos, A. C., Velázquez-Camarillo, A. A., \& Bautista-Díaz, M. L. (2019). Calidad de vida, sintomatología de ansiedad y depresión en personas con $\mathrm{VIH}$ : El papel del psicólogo de la salud. Educación y Salud Boletín Científico Instituto de Ciencias de la Salud Universidad Autónoma del Estado de Hidalgo, 8(15), 80-91. https:// doi.org/10.29057/icsa.v8i15.4829

Centro Nacional para la Prevención y Control del VIH/Sida (2019). Vigilancia epidemiológica de casos de VIH/SIDA en México. Registro Nacional de Casos de SIDA, actualización al 11 de noviembre de 2019. https://www.gob.mx/cms/ uploads/attachment/file/513720/RN_D_a_Mundial_ sida_2019.pdf

Chamorro, L., \& Betanco, N. (2019). Conocimientos, actitudes y prácticas de $\mathrm{VIH} / \mathrm{SIDA}$ en los trabajadores de la salud que laboran para el MINSA en el municipio de Somotillo. Tesis doctoral. Universidad Nacional Autónoma de Nicaragua. http://riul.unanleon.edu.ni:8080/jspui/ handle/123456789/7073

Chávez, M. A., Zapata, J., Petrzelová, J., \& Villanueva, G. (2018). La diversidad sexual y sus representaciones en la juventud. Psicogente, 21(39), 62-74. https://doi.org/10.17081/ psico.21.39.2822

Conejeros, I., Emig, H., Ferrer, L., Cabieses, B., \& Acosta, R. C. (2010). Conocimientos, actitudes y percepciones de enfermeros y estudiantes de enfermería hacia el VIH/ SIDA. Investigación y Educación en Enfermería, 28(3), 345354. https://revistas.udea.edu.co/index.php/iee/article/ view/7589

Damulira, C., Mukasa, M. N., Byansi, W., Nabunya, P., Kivumbi, A., Namatovu, P., Namuwonge, F., Dvalishvili, D., Sensoy Bahar, O., \& Ssewamala, F. M. (2019). Examining the relationship of social support and family cohesion on ART adherence among HIV-positive adolescents in southern Uganda: Baseline findings. Vulnerable Children \& Youth Studies, 14(2), 181-190. https://doi.org/10.1080/17450 128.2019.1576960

DiClemente, R., \& Wingood, G. (1995). A randomized controlled trial of an hiv sexual risk reduction intervention for young african-american women. Journal of the American Medical Association, 274(16), 1271-1276. https://doi. org/10.1001/jama.274.16.1271

Ebrahimi, F., Sindarreh, S., \& Nasirian, M. (2020). Attitudes and Practice of Health Care Workers about Human Immunodeficiency Virus in Isfahan, Iran. Iranian Journal of Nursing \& Midwifery Research, 25(2), 111-116. https:// doi.org/10.4103/ijnmr.IJNMR_67_19

Elshiekh, H. F., Hoving, C., \& De Vries, H. (2020). Exploring determinants of condom use among university students in Sudan. Archives of Sexual Behavior, 49, 1379-1391. https://doi.org/10.1007/s10508-019-01564-2

Fernández, B., Colunga, C., González, M., Aranada, M. I., Riego, N. A., Sánchez, A., Enríquez, C., \& Balderrama, J. (2013). Nivel de conocimientos sobre sexualidad en estudiantes de carreras de Ciencias de la Salud de la Universidad Veracruzana. Revista UniverSalud, 9(17), 12-18. https:// www.uv.mx/msp/files/2012/12/UniverSalud-17.pdf

Feyissa, G. T., Lockwood, C., Woldie, M., \& Munn, Z. (2019). Reducing HIV-related stigma and discrimination in healthcare settings: A systematic review of quantitative evidence. PLOS ONE, 14(1), 1-23. https://doi. org/10.1371/journal.pone.0211298

Finkelstein-Fox, L., Park, C., \& Kalichman, S. (2020). Health benefits of positive reappraisal coping among people living with HIV/AIDS: A systematic review. Health Psychology Review, 14(3), 394-426. https://doi.org/10.1 $080 / 17437199.2019 .1641424$

Flores-Cano, O., Reyes-Lagunes, I., Rivera-Aragón, S., \& Mansilla-Corona, R. (2020). Bienestar subjetivo y su estudio con redes semánticas en análisis lineal y no lineal. Interdisciplina, 8(20), 153-167. https://doi. org/10.22201/ceiich.24485705e.2019.18.71207

Granados, R. (2019). Validación psicométrica de la Escala de Valoración de Riesgo de Violencia en Adolescentes. Archivos de Criminología, Seguridad Privada y Criminalística, 12(6), 107-121.

Gredig, D., Le Breton, M., Granados, I., \& Solís, V. (2020). Predictores del uso del condón en hombres que tienen relaciones sexuales con hombres en Costa Rica: Comprobación del modelo de información, motivación y habilidades conductuales. RICS Revista Iberoamericana de las Ciencias de la Salud, 9(17), 25-54. https://doi. org/10.23913/rics.v9i17.83

Guerra, M., Aldana, E., \& Rojas, I. (2020). Conocimiento sobre prevención de infecciones de transmisión sexual en adolescentes inmigrantes habitantes en Soledad-Atlántico 2018-2019. Identidad Bolivariana, 4(1), 5-15. https://doi. 
org/10.37611/IB4ol15-15

Kerlinger, F., \& Lee, H. (2002). Investigación del comportamiento. Métodos de investigación en ciencias sociales. México: McGraw Hill.

Llibre, J. M., Fuster-Ruizdeapodaca, M. J., Rivero, A., \& Fernández, E. (2018). Cuidados clínicos del paciente con VIH. Enfermedades Infecciosas y Microbiología Clínica, 36, 40-44. https://doi.org/https://doi.org/10.1016/S0213005X(18)30246-5

Mak, W. W. S., Cheng, S. S. Y., Law, R. W., Cheng, W. W. L., \& Chan, F. (2015). Reducing HIV-related stigma among health-care professionals: A game-based experiential approach. AIDS Care, 27(7), 855-859. https://doi.org/10.1080/0954012 1.2015 .1007113

Marshall, A., Brewington, K., Kathryn, A., Haynes, T., \& Zaller, N. (2017). Measuring HIV-related stigma among healthcare providers: A systematic review. AIDS Care, 29(11), 13371345. https://doi.org/10.1080/09540121.2017.1338 654

Mascolo, P., Michelena, A., Monestiroli, D., Montes, I., Martínez, M., \& Rodríguez-Morales, A. (2011). Conocimientos, actitudes y percepciones sobre VIH/SIDA e infecciones de transmisión sexual en estudiantes ingresados a odontología y medicina de una universidad venezolana. Revista Médica de Risaralda, 17(2), 70-76. https://doi. org/10.22517/25395203.7585

Nguyen, M. X., Go, V. F., Bui, Q. X., Gaynes, B. N., \& Pence, B. W. (2019). Perceived need, barriers to and facilitators of mental health care among HIV-infected PWID in Hanoi, Vietnam: A qualitative study. Harm Reduction Journal, 16(1), 1-9. https://doi.org/10.1186/s12954-019-03498

Novales-Castro, X. J., Amato, D., \& Hurtado, M. M. T. (2019). Conductas sexuales en estudiantes de licenciatura del área de la salud. Revista Electrónica de Psicología Iztacala, 22(3), 2658-2670. http://www.revistas.unam.mx/index. $\mathrm{php} / \mathrm{repi} /$ article/view/70934

Peer, N., de Villiers, A., Jonathan, D., Kalombo, C., \& Kengne, A.P. (2020). Care and management of a double burden of chronic diseases: Experiences of patients and perceptions of their healthcare providers. PLOS ONE, 15(7), 1-14. https://doi.org/10.1371/journal.pone.0235710

Pineda-Roa, C. A., \& Campo-Arias, A. (2018). Estudio psicométrico de una escala de actitud frente al VIH-sida en estudiantes de psicología de dos escuelas colombianas. Duazary, 15(1). https://doi.org/10.21676/2389783X.2022

Pittenger, A. L., Goodroad, B., Nicol, M., Durgin, E. N., Eveland, L., Kaiser, R., Benbenek, M., Benson, N., \& Rybicki, S. (2019). Interprofessional Education Within a Pilot HIVCare Elective Course. American Journal of Pharmaceutical Education, 83(10), 2053-2058. https://doi.org/10.5688/ ajpe7402Reyes-Lagunes, I. (1993). Redes semánticas para la construcción de instrumentos. Revista de Psicología Social y Personalidad, 9(1), 83-99.

Robles, S., \& Díaz-Loving, R. (2011). Validación de la Encuesta Estudiantil sobre salud sexual (EESS). México: UNAM, Facultad de Estudios Superiores Iztacala.

Rodríguez, L. (2019). Percepción y actitudes del alumnado de trabajo social mexicano hacia

el VIH. En C. Praca, A. Fontenelle \& J. Peixoto. Tendências e tecnologias na prom o ção da

saúde nos espaços educacionais (pp. 11-36). EdUECE.

Sharma, S. K., Mudgal, S. K., Thakur, K., Gaur, R., \& Aggarwal, P. (2020). Lifestyle behavior of budding health care professionals: A cross-sectional descriptive study. Journal of family medicine and primary care, 9(7), 3525-3531. https://doi.org/10.4103/jfmpc.jfmpc_491_20

Shahar, E., Maor, C., \& Moshe-Eilon, Y. (2020). Medical personnel knowledge and stigmatic attitude toward HIV patients in a high-income country. AIDS Care, 32, 1023-1029. https:// doi.org/10.1080/09540121.2020.1734172

Shey, N. D., Dzemo, K. O., Siysi, V. V., Ekobo, A. S., \& Jelil, N. A. (2020). Quality of life of HIV patients on highly active antiretroviral therapy: A scoping review. Journal of Public Health and Epidemiology, 12(1), 63-73. https://doi. org/10.5897/JPHE2019.1148

Sigüenza, C., \& Solano, N. (2017). Conocimientos y actitudes sobre VIH/SIDA en el personal de salud del hospital Vicente Corral Moscoso. 2016. Tesis de licenciatura. Universidad de Cuenca. http://dspace.ucuenca.edu. ec/handle/123456789/28016Uribe, A., \& Orcasita, L. (2011). Evaluación de conocimientos, actitudes, susceptibilidad y autoeficacia frente al $\mathrm{VIH} / \mathrm{sida}$ en profesionales de la salud. Avances en enfermería, 29(2), 271-284. https://revistas.unal.edu.co/index.php/ avenferm/article/view/35821

Valdez, E., Vargas, A., \& Apaza, M. (2010). Conocimientos, actitudes y prácticas del personal de salud sobre el VIH/ SIDA de Cochabamba, estudio comparado, 12 años después. Revista Médica (Cochabamba), 21, 53-63.

Varas-Díaz, N., Rivera-Segarra, E., Neilands, T. B., Pedrogo, Y., Carminelli-Corretjer, P., Tollinchi, N., Torres, E., Soto Del Valle, Y., Rivera Díaz, M., \& Ortiz, N. (2019). HIV/AIDS and intersectional stigmas: Examining stigma related behaviours among medical students during service delivery. Global Public Health, 14(11), 1598-1611. https:// doi.org/10.1080/17441692.2019.1633378

Wagner, A. C., McShane, K. E., Hart, T. A., \& Margolese, S. (2016). A focus group qualitative study of HIV stigma in the Canadian healthcare system. Canadian Journal of Human Sexuality, 25(1), 61-71. https://doi.org/10.3138/ cjhs.251-A6

Wondemagegn, F., \& Berkessa, T. (2020). High level risky sexual behavior among persons living with HIV in the urban setting of the highest HIV prevalent areas in Ethiopia: Implications for interventions. PLOS ONE, 15(11), 1-14. https://doi.org/10.1371/journal.pone.0242701

Wray, T., Chan, P., \& Simpanen, E., (2020). Longitudinal effects of home-based HIV self-testing on well-being and health empowerment among men who have sex with men (MSM) in the United States. AIDS Care, 32(2), 148-154, https://doi.org/10.1080/09540121.2019.1622636 


\section{Meta-Análisis del Artículo}


Artículo Empírico | Conocimientos y percepción de estudiantes...| Sánchez-Medina, et al.

\section{Dimensión Cuantitativa}

\section{Perfil de Evaluación entre pares}
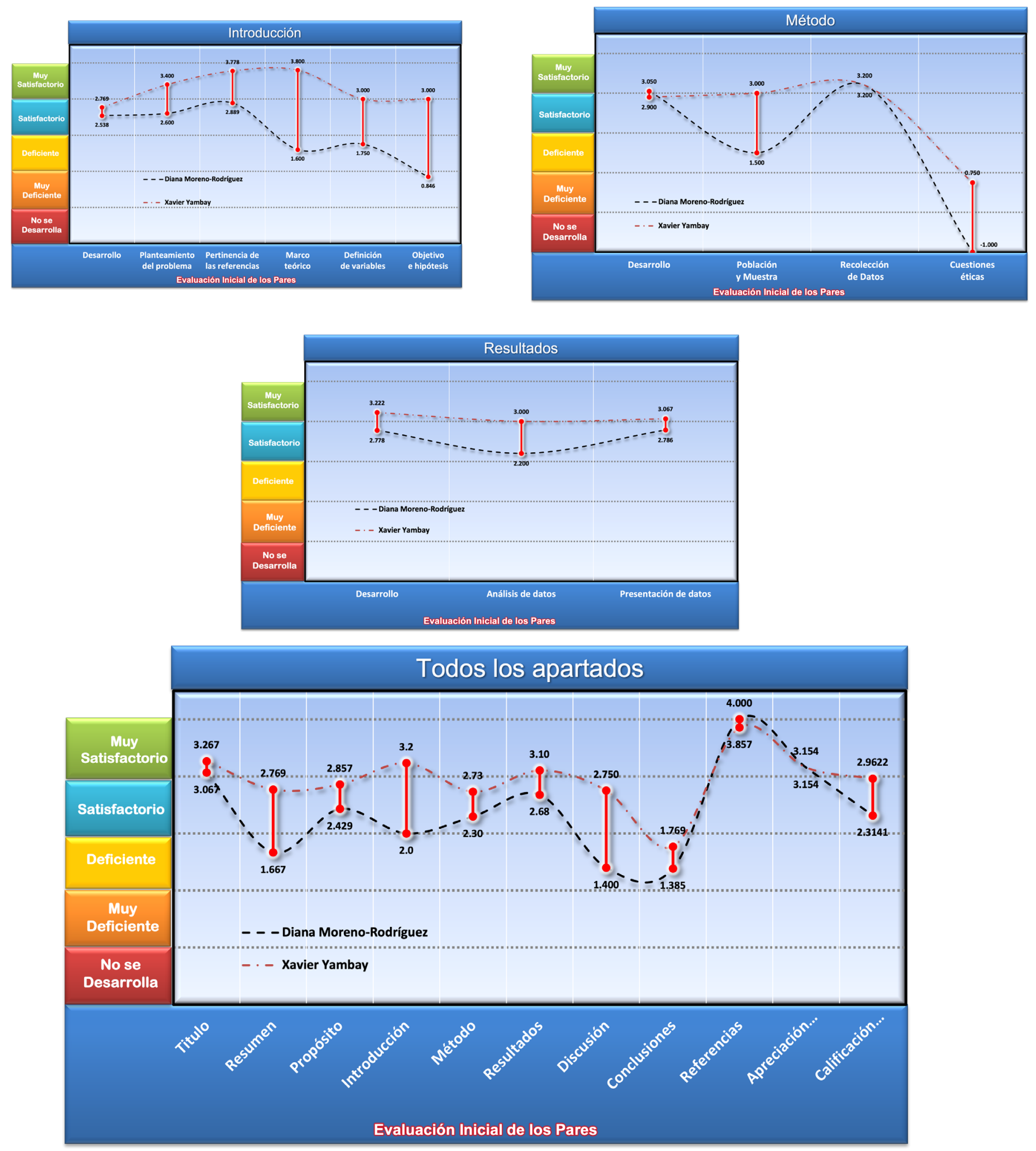


\section{Índice de Concordancia}

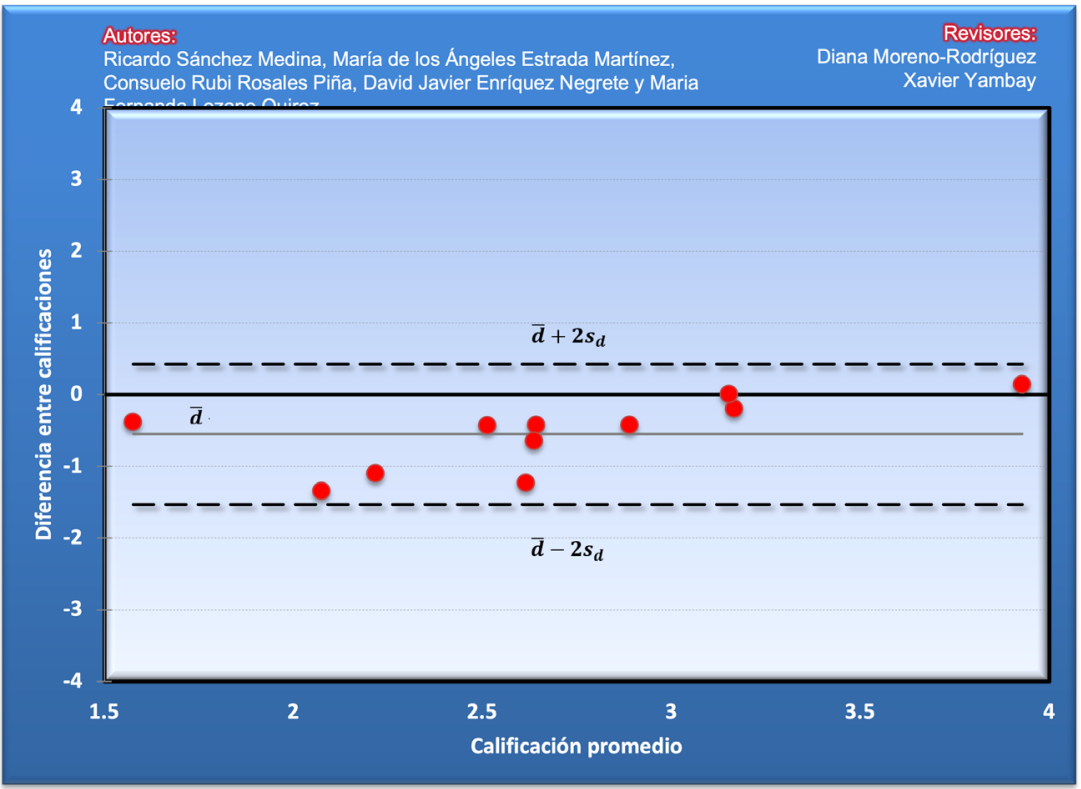

Índice de Acuerdo

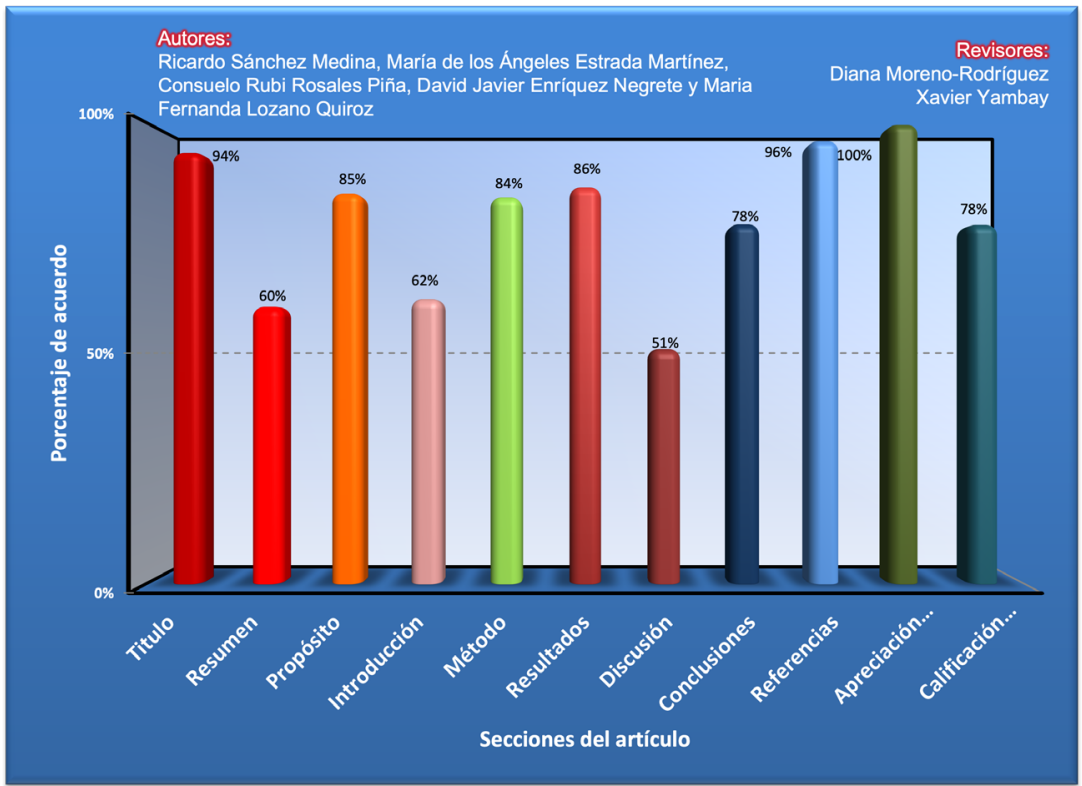




\begin{tabular}{|c|c|}
\hline Revisor 1 & Revisor 2 \\
\hline Diana Moreno Rodríguez & Xavier Yambay \\
\hline \multicolumn{2}{|c|}{ Título/Autoría } \\
\hline $\begin{array}{l}\text { El título tiene problemas de construcción gramatical. CO- } \\
\text { NOCIMIENTOS Y PERCEPCIÓN DE ESTUDIANTES } \\
\text { DE PSICOLOGIIA SOBRE EL TRABAJO PROFESIO- } \\
\text { NAL EN PERSONAS CON VIH El trabajo profesional es } \\
\text { con personas, no de personas }\end{array}$ & \\
\hline \multicolumn{2}{|c|}{ Resumen } \\
\hline $\begin{array}{l}\text { Se recomienda que el resumen en español siga la se- } \\
\text { cuencia mencionada en los puntos } 3,4,5 \text { y } 6 \text {. El conte- } \\
\text { nido del primer párrafo de ninguna manera justifica lo } \\
\text { que se va hacer, hay una desvinculación ente el primer } \\
\text { párrafo con lo objetivos señalados. Por otra parte, con } \\
\text { relación al resumen en ingles, se recomienda se haga la } \\
\text { traducción con un conocedor del idioma inglés y que } \\
\text { no sea una traducción de traductor de google. El pri- } \\
\text { mer párrafo no se entiende. la palabra transmit no se } \\
\text { usa para expresar lo que se sea. }\end{array}$ & \\
\hline \multicolumn{2}{|c|}{ Próposito del Estudio } \\
\hline $\begin{array}{l}\text { Se recomienda revisar los argumentos que dan origen } \\
\text { al estudio, existen confusiones e ideas incompletas que } \\
\text { dificultan la lectura }\end{array}$ & \\
\hline \multicolumn{2}{|c|}{ Introducción } \\
\hline
\end{tabular}




\begin{tabular}{|l|}
\hline Revisor 1 \\
\hline El segundo párrafo de la introducción es confuso, pa- \\
labras que no corresponden. contrastar los efectos???, \\
adherencia no farmacológica, cuál es ese tipo de adhe- \\
rencia?, deben conocer en qué consiste el virus?, qué se \\
quiere decir con esto?,. serios problemas de redacción. \\
Párrafo 4 de la introducción igualmente se hacen una \\
serie de afirmaciones, sin vinculación con el último pá- \\
rrafo. Igualmente se hace una afirmación en el sentido \\
de que el psicólogo debe contar con conocimientos,, \\
habilidades y actitudes positivas, pero no se dice hacia \\
qué?, por lo que es una idea incompleta. En el párrafo \\
5 no es claro hacia quien se dirige el personal de sa- \\
lud e.g. permite mejorar su calidad de vida de quien? \\
Párrafo 7 Permite la aplicación de ciertas técnicas, en \\
ciencia no se puede ser tan ambiguo, se sabe cuales son \\
se deben mencionar. En otro espacio se habla de cono- \\
cimientos y habilidades que debe poseer el psicólogo, \\
conocimientos sobre qué habilidades cómo cuales y \\
con que fin? igualmente percepción acerca de qué. No \\
esta justificada la técnica de RSNM por qué usarla, ade- \\
más de que no se mencionan su significado, cuando se \\
alude por primera vez.
\end{tabular}

Revisor 2

Al describir el objetivo 1, la frase es redundante, se sugiere utilizar otros verbos, por ejemplo: explorar, identificar, etc. También la palabra conocimiento debe ir en singular y no en plural. Además, se entiende que el uso del condón sería en relaciones sexuales, se sugiere eliminar esta frase que está demás.

\section{Método}

A lo largo de toda la introducción se menciona la importancia de que el psicólogo posea habilidades (cuáles no se dice), ni tampoco estas se evalúan en el estudio.

Debería incluirse el tipo de investigación, diseño y enfoque de la misma. Se sugiere detallar las consideraciones éticas (consentimiento informado, riesgos potenciales, etc)

\section{Resultados}

Algunas de las afirmaciones señaladas son por demás ambiguas, cuando se dice que los estudiantes tienen un nivel alto, moderado o bajo de conocimiento, por que usar estos términos, y cual es el criterio para hacer tal aseveración. Nuevamente qué es poseer poco este tipo de creencias? nuevamente la ambigüedad y confusión para referirse a las creencias. En la Tabla 2 , es necesario que los autores hagan una descripción más precisa de lo representado en ésta, una correlación siempre debe referirse en términos de su dirección (positiva o negativa) e intensidad (media, baja o alta), al respeto hay literatura que así lo señala y para los valores específicos también en dicha literatura se menciona. Algo que de alguna manera puede ser problema es la mayoría de las correlaciones encontradas son muy bajas.

Sin sugerencias 


\begin{tabular}{|c|c|}
\hline Revisor 1 & Revisor 2 \\
\hline \multicolumn{2}{|c|}{ Discusión } \\
\hline $\begin{array}{l}\text { Nuevamente se hacen afirmaciones sin ningún susten- } \\
\text { to y/o preguntas que no se responden 1.- Se afirma que } \\
\text { es necesario que los estudiantes de psicología tengan } \\
100 \% \text { en sus conocimientos, por qué no se dice. 2.- Por } \\
\text { que es importante este tipo de conocimientos, cuando } \\
\text { se pretende trabajar con pacientes con VIH, que im- } \\
\text { plicaciones tiene esto. Por otra parte se deja de lado, } \\
\text { el supuesto propósito del vínculo entre conocimientos, } \\
\text { habilidades y actitudes, cuando se trabaja en el campo } \\
\text { profesional con pacientes O+. En la introducción se ha- } \\
\text { bla de habilidades, esta variable no se evaluó,. De qué } \\
\text { sirve diseñar estrategias para que los estudiantes, usen } \\
\text { condón, que tiene que ver con el trabajo profesional del } \\
\text { VIH. Afirman que el trabajo de psicólogo es promover } \\
\text { estilos saludables con pacientes O+, cuestión que está } \\
\text { vinculada con la esencia del trabajo o investigación. Si } \\
\text { es así deberá dedicársele un espacio, sino queda fuera } \\
\text { de contexto sin ningún sustento. Limitaciones del estu- } \\
\text { dio no está desarrollado Implicaciones del trabajo No } \\
\text { esta desarrollado Direcciones futuras del estudio No } \\
\text { está desarrollado. }\end{array}$ & Incluir el detalle de las limitaciones del estudio \\
\hline \multicolumn{2}{|c|}{\begin{tabular}{|l|l} 
& Conclusiones \\
\end{tabular}} \\
\hline Es importante poner atención a los puntos $10,11,12$ y 13 , & $\begin{array}{l}\text { Señalar los aportes o contribución de la investigación } \\
\text { para la sociedad. Proponer qué investigaciones se pue- } \\
\text { den realizar a futuro tomando como punto de inicio su } \\
\text { estudio. }\end{array}$ \\
\hline \multicolumn{2}{|c|}{ Referencias } \\
\hline Sin comentarios & Sin sugerencias \\
\hline
\end{tabular}




\section{Historia del Proceso EDITORIAL}

